\title{
From EXO-200 to nEXO
}

\author{
Andrea Pocar*t \\ Amherst Center for Fundamental Interactions and Physics Department, University of \\ Massachusetts, Amherst, Massachusetts 01003, USA \\ Physics Division, Lawrence Livermore National Laboratory, Livermore, California 94550, USA \\ E-mail: pocar@umass.edu
}

The search for neutrino-less double beta decay $(0 v \mathrm{DBD})$ is currently undertaken with experiments using 100's kg of candidate isotope in ultra low background detectors housed deep underground. We report on the recent data from the EXO-200 experiment on $2 v$ and $0 v \mathrm{DBD}$ of ${ }^{136} \mathrm{Xe}$, and provide a summary of "lessons learned". The physics case is made for a next generation, tonne-scale nEXO project based on the EXO-200 performance, with added proposed technical innovations. An overview of the R\&D activities currently under way to make nEXO possible is presented, along with a brief mention to the future of EXO-200.

XVI International Workshop on Neutrino Telescopes,

2-6 March 2015

Palazzo Franchetti â Istituto Veneto, Venice, Italy

\footnotetext{
${ }^{*}$ Speaker.

† for the EXO-200 and nEXO collaborations.
} 


\section{Introduction}

The search for neutrino-less double beta decay $(0 v \mathrm{DBD})$ is one of the few, if not the only practical way to investigate whether neutrinos are Majorana particles, i.e. their own antiparticles [1]. It is the simultaneous decay of two neutrons (protons) of a nucleus into two protons (neutrons) with the emission of two electrons (positrons, or the absorption of capture electrons) but no neutrinos. $0 v \mathrm{DBD}$ violates lepton number by two units, a global symmetry so far never observed to be broken. It can be driven by a virtual exchange of light massive neutrinos, a description that requires the minimum perturbation to the Standard Model. In this framework, its half life is related to an effective neutrino mass as:

$$
\left(T_{1 / 2}^{0 v}\right)^{-1}=G(Q, Z) \cdot|M|^{2} \cdot\left\langle m_{\beta \beta}\right\rangle^{2}
$$

where $G$ is a calculable phase space factor, $M$ is an $O(1)$ nuclear matrix element connecting initial and final nuclear states, and $\left\langle m_{\beta \beta}\right\rangle=\left|\sum_{j=1}^{3} U_{e j}^{2} m_{j}\right|$, a coherent sum of neutrino masses $\left(U_{e j}\right.$ are complex elements of the PMNS unitary neutrino mixing matrix). Other mechanisms are possible that could drive $0 v \mathrm{DBD}$. Irrespective of the fundamental physics at play, the observation of $0 \mathrm{vDBD}$ implies that neutrinos are massive Majorana particles [2].

The search for $0 v \mathrm{DBD}$ is currently undertaken with experiments using 100's kg of candidate isotope in ultra low background detetors housed deep underground. The state of the art of these reaches is summarised in Fig. 1, where the lower limits on the half lives of ${ }^{76} \mathrm{Ge}$ (GERDA [3]), and ${ }^{136} \mathrm{Xe}$ (KamLAND-Zen [4], and EXO-200 [5]) are shown and compared by converting them into upper limits of $\left\langle m_{\beta \beta}\right\rangle$. Continuous running of these experiments, along with others scheduled to come online in the near future (e.g. CUORE [7], SNO+ [8] using ${ }^{130} \mathrm{Te}$ ) promises to push the half life sensitivity to $\sim 10^{26}$ years.

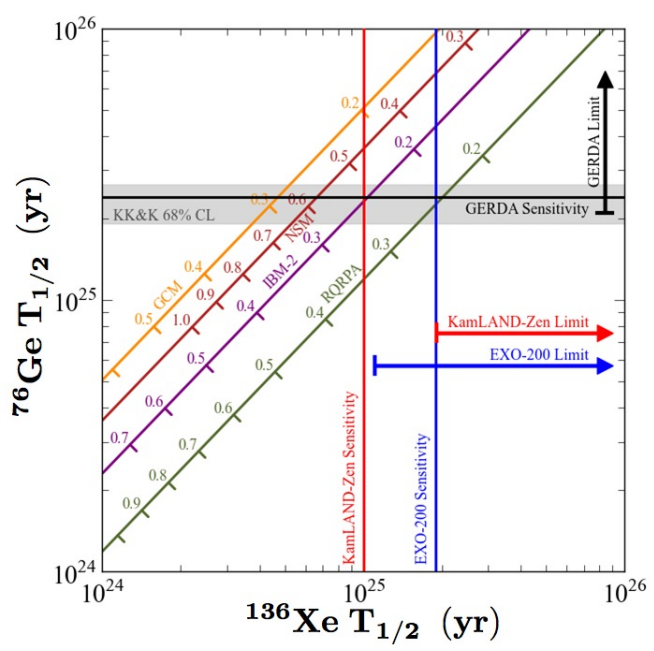

Figure 1: Current best limits and sensitivity for the half life of $0 v \mathrm{DBD}$ obtained with ${ }^{136} \mathrm{Xe}$ and ${ }^{76} \mathrm{Ge}$, and corresponding upper limits on the effective Majorana neutrino mass [3, 4, 5]. These results are in very strong tension with a claim of observation of $0 v \mathrm{DBD}$ in ${ }^{76} \mathrm{Ge}[6]$. 


\section{EXO-200}

The Enriched Xenon Observatory (EXO) program started fifteen years ago with the goal to search for $0 v \mathrm{DBD}$ of ${ }^{136} \mathrm{Xe}$ with Time Projection Chambers (TPCs) filled with enriched xenon of ever increasing sensitivity. The program's first experiment, EXO-200, uses $200 \mathrm{~kg}$ of enriched liquid xenon (eLXe) and has been running since 2011 at the WIPP underground site in Carlsbad, New Mexico. A larger experiment, nEXO, based on the EXO-200 experience and employing 5 tonnes of LXe is now being designed with $\sim 100 \times$ the half life sensitivity of EXO-200.

EXO-200 has made the first measurement of the $2 v$ DBD of ${ }^{136} \mathrm{Xe}$ in 2011 [9]. This is the longest-lived double beta decaying isotope among a handful with $Q>2 \mathrm{MeV}$, and the last one to be measured $\left(Q\left({ }^{136} \mathrm{Xe}\right)=2.458 \mathrm{MeV}\right.$ [11]). However, with a half life of $(2.165 \pm 0.016($ stat $) \pm$ $0.059($ sys $)) \times 10^{21}$ years measured by EXO-200 in 2013 [10], it is as yet the most precisely measured. The recent history of ${ }^{136} \mathrm{Xe} 2 v$ DBD measurements is summarised in Fig. 2.

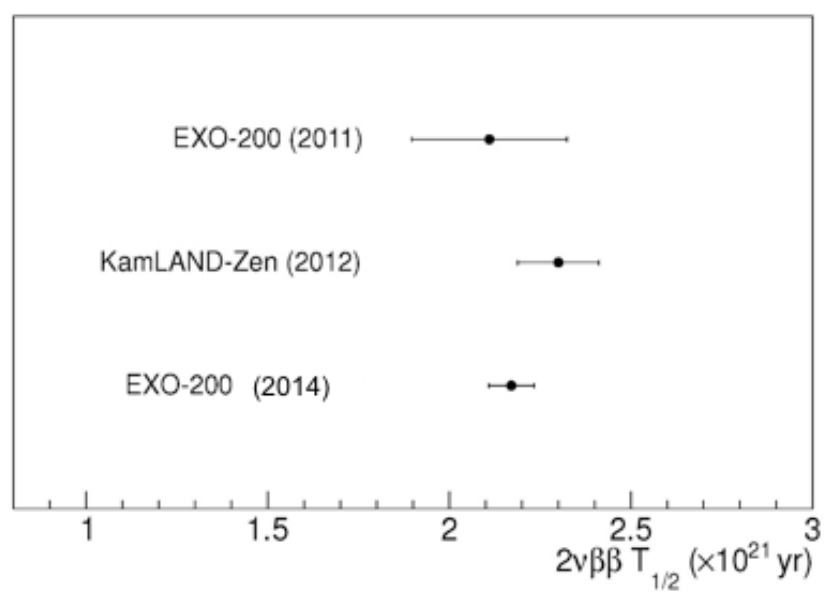

Figure 2: The evolution of the measurements of 2vDBD by EXO-200 [9, 10] and KamLAND-Zen [4].

EXO-200 [12] makes use of the property of TPCs to record and distinguish the energy and position of individual energy deposition in the detector (a view of the TPC during assembly is shown in Fig. 3, left). A vast majority of DBD events are confined to a small volume of the detector (MeV electrons travel only a few $\mathrm{mm}$ in LXe) and are classified as single-site. EXO-200 collects ionization charge on crossed wires at both ends of a cylindrical volume divided in the middle by a cathode mesh at -HV. Wires have $3 \mathrm{~mm}$ pitch, with three ganged into one read out channel. Adjacent channels thus have a $9 \mathrm{~mm}$ pitch, much larger than the typical size of DBD ionization clouds. In addition to ionization, EXO-200 detects LXe scintillation light vie $~ 500$ silicon large area avalanche photodiodes (LAAPDs) installed behind the crossed wire planes at both ends of the cylindrical xenon vessel. The event-by-event combination of ionization and scintillation is crucial is reducing the energy resolution (see Fig. 3, right). The majority of the background comes from $\mathrm{MeV}$-scale $\gamma$-rays. At this energy, photons are most likely to Compton scatter, with a large fraction being hence classified as multi-site. The effectiveness of this discrimination is illustrated in Fig. 4. When analysed from the purely calorimetric point of view, this figure also shows that the background index (defined from the $\pm 2 \sigma$ ROI interval at the endpoint, $\frac{\sigma}{E}(Q)=1.53 \%$ ) for single-site events is $(1.7 \pm 0.2) \times 10^{-3}$ events $/ \mathrm{kg} / \mathrm{keV} / \mathrm{yr}$, among the best in the field. 

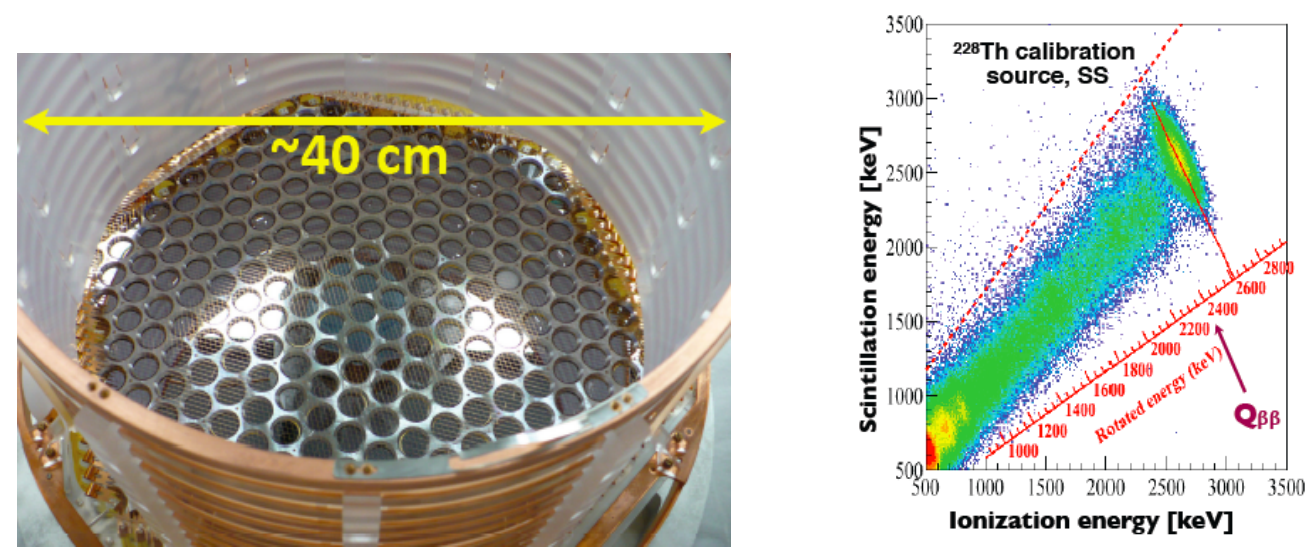

Figure 3: Left: One of two symmetric TPCs constituting the EXO-200 inner detector, during assembly at Stanford. Right: use of event-by-event anti-correlation of the amplitude of the ionization and scintillation signals to define the preferred energy axis with optimal energy resolution. The plot shows data from a ${ }^{228} \mathrm{Th}$ source with the signature $2,615 \mathrm{keV} \gamma$ ray.

\section{EXO-200: Lessons learned}

The EXO-200 measurements show that xenon is a very promising isotope for multi-tonne DBD searches with a very large detector. In addition to the topology recognition described above, compared to competing isotopes, it is cheaper and more straight-forward to enrich ${ }^{1}$, can be used in gas and liquid detectors with large unsegmented central volumes shielded from the radioactivity of passive structures, and allows for continuous recirculation and purification from most intrinsic radioactivity and from electronegative impurities. A large enriched isotope stockpile can be reused in different detectors, should the need arise to abate backgrounds further or run next generation detectors, perhaps to follow up on signal hints.

A modest improvement in energy resolution from EXO-200, $\frac{\sigma}{E}(Q) \sim 1 \%$, is sufficient to contain the irreducible background from $2 v$ DBD into the $0 v$ DBD search window for a multi-tonne experiment to well below 1 count in 10 years. As unsegmented detectors get larger, the self-shielding property of dense LXe for $\sim \mathrm{MeV}$ photons becomes an ever so powerful background discrimination tool. For reference, $2.5 \mathrm{MeV} \gamma$-rays have an attenuation length of $8.5 \mathrm{~cm}$ in $\mathrm{LXe}$, a substantial fraction of the linear dimension of the EXO-200 TPC (a cylinder of roughly $40 \mathrm{~cm}$ diameter and $40 \mathrm{~cm}$ length). Finally, xenon is unique as it allows one to detect the appearance of ${ }^{136} \mathrm{Ba}$ following double beta decays thus potentially suppressing all non-DBD backgrounds ("barium tagging") [13].

In particular, EXO-200 has also demonstrated:

- measured backgrounds are consistent with radio-assays, making predictions possible in the design and construction phases [14].

- superb LXe purity from electronegative contaminants, measured by stable electron life time $>3$ ms over a 2-year period.

- the power of LXe standoff in suppressing $\gamma$ ray background in a monolithic, homogeneous detector.

\footnotetext{
${ }^{1}$ All isotopes under consideration for tonne-scale experiments are less than $10 \%$ naturally abundant and require enrichment, with the possible exception of ${ }^{130} \mathrm{Te}$, which has $\sim 30 \%$ natural abundance.
} 


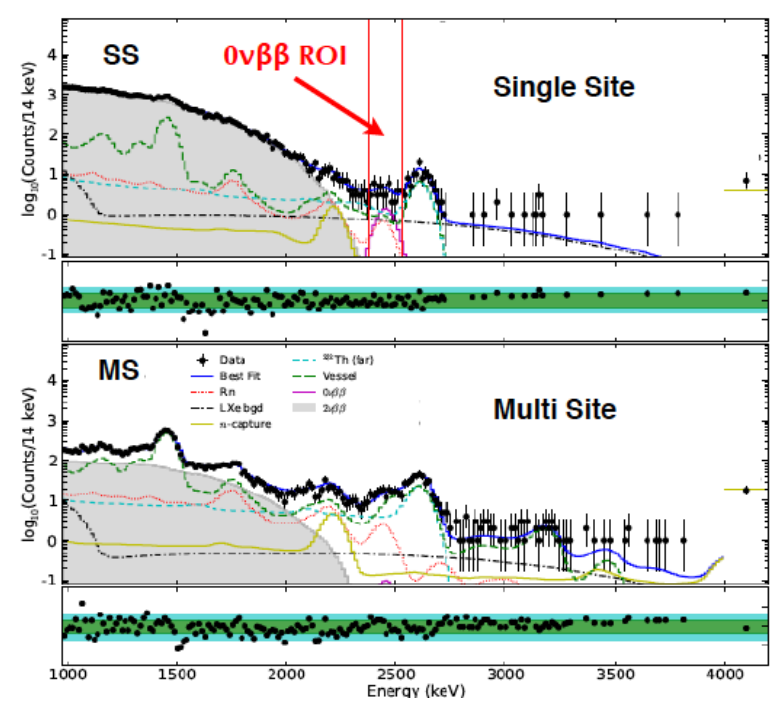

Figure 4: Left: Right: The EXO-200 energy spectrum for a $\sim 1.5$ year long low background run, divided into single- and multi-site events SS, ad MS, respectively), allowing for a effective discrimination of multiplescattering $\gamma$-ray background [5].

- powerful $\gamma / \beta$ discrimination, and almost perfect $\alpha / \beta$ identification [15].

- first extensive use of un-encapsulated silicon LAAPDs in lieu of more traditional photomultiplier tubes [16].

- use of bornze, photo etched wire and mesh electrodes, and spring contacts.

- home-made, He leak tight epoxy feedthroughs for kapton flat cables.

- electrical cryogenic design free of solder joints or connectors.

- cryogenic scheme employing a many-tonne, perfluoroether thermal bath around the xenon chamber.

The enriched xenon was successfully recovered operating the detector remotely after the mine evacuation.

EXO-200 is currently recommissioning the underground experimental installation following two incidents in February 2014 that have interrupted access to WIPP and forced us to stop data taking and warm up the detector. Both hardware and analysis upgrades are being readied. The enriched xenon was successfully recovered operating the detector remotely after the mine evacuation. One hardware novelty is the new front end electronics to substantially reduce the coherent noise component on the LAAPD channels, which should allow EXO-200 to reach 1\% energy resolution at $Q$. The other is the addition of a radon-scrubbing "deradonator" to supply radon-free air to the air gap surrounding the EXO-200 cryostat. According the our background model [5], up to 10\% of the background in the $0 v \mathrm{DBD}$ endpoint ROI is attributable to $2449 \mathrm{keV}$ gamma rays from ${ }^{214} \mathrm{Bi}$ (a radon daughter) decays in said air gap. EXO-200 is scheduled to take another 2-3 years of data, with a projected sensitivity to $0 v \mathrm{DBD}$ of ${ }^{136} \mathrm{Xe}$ in excess of $5 \times 10^{25}$ years 


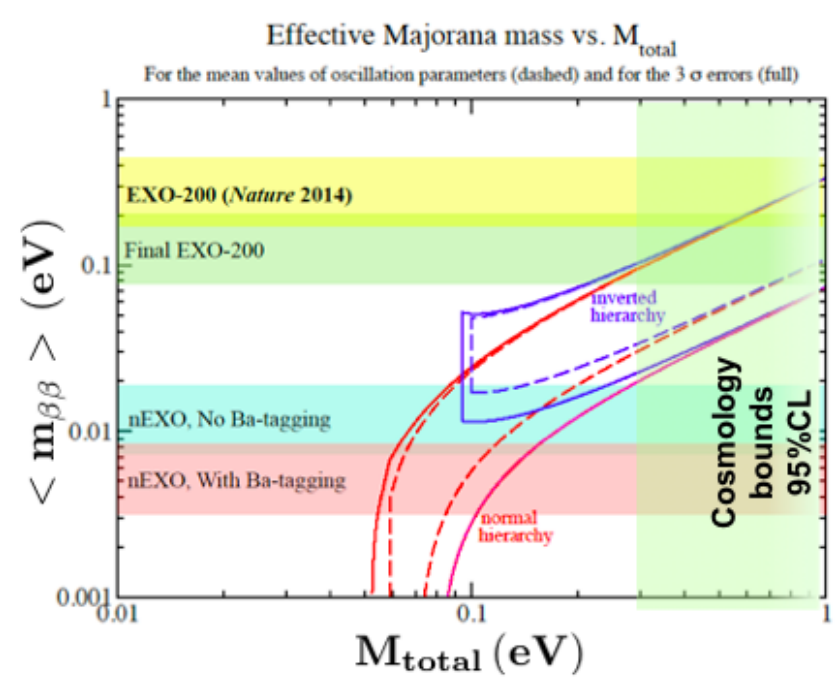

Figure 5: Majorana neutrino mass sensitivity versus the smallest neutrino masses for EXO-200 (present and projected) and nEXO (with and without Ba tagging). For EXO-200, the extrapolated sensitivity presumes a further 2-year run with upgraded electronics for the scintillation detector and low radon air around the cryostat. The red and blue bands come from neutrino oscillation parameters. Five years of data from nEXO allow to cover the inverted neutrino mass hierarchy for most nuclear matrix elements.

\section{4. nEXO}

The $\mathrm{nEXO}$ Collaboration ${ }^{2}$ is performing R\&D towards a detector with half life sensitivity to $0 v \mathrm{DBD}$ of ${ }^{136} \mathrm{Xe} 6 \times 10^{27}$ years using five tonnes of enriched LXe $(\times 25$ the amount of xenon used for EXO-200). It is important to point out that EXO-200 was itself a $\times 20$ scale-up from the previous Gotthard gaseous xenon TPCs for DBD [17]. Low background, dual phase xenon TPC have also proven such scale-ups possible, and are too designing tonne-scale detectors [18, 19, 20]. An experiment with $>100 \times$ the sensitivity of current experiments, nEXO promises to completely cover the inverted neutrino mass hierarchy (for most nuclear matrix elements, with $\left\langle m_{\beta \beta}\right\rangle=15-25$ $\mathrm{meV}$ ) in five years of running (see Fig. 5). R\&D towards a nEXO upgrade making use of a barium tagging system is also being carried out in parallel.

Rooted in the EXO-200 design and experience, $\mathrm{nEXO}$ displays some notable differences from its predecessor. The xenon is contained in a $\sim 1.3 \times 1.3 \mathrm{~m}$ right cylinder, with one single drift volume. This layout puts more challenges on the HV supply but eliminates anything other than xenon from the center of the detector. In fact, the definition of tonne-scale xenon volume is one of the key design criteria. The detector is much larger than the attenuation length of $2.5 \mathrm{MeV}$ photons, creating the conditions for an extremely low $\gamma$-ray background in the bulk of the active detector.

It is worthwhile to underline how energy resolution is not the only key detector parameter for a successful $0 v$ DBD search. nEXO will take full advantage of the "standoff distance" variable, i.e. the distance to the closest non-Xe structure in the detector, which allows to discriminate between external background and uniformly distributed DBD events. The power of this technique critically

\footnotetext{
${ }^{2}$ The nEXO Collaboration includes scientists from the USA (Alabama, Brookhaven, Drexel, Duke, Illinois, Indiana, Livermore, Oak Ridge, SLAC, South Dakota, Stanford, Stony Brook, UMass), Canada (Carleton, Laurentian, TRIUMF), Switzerland (Bern), Germany (Erlangen, Munich), China (IHEP), Russia (ITEP), South Korea ()
} 
pivots on measuring the background inside the detector and its attenuation from the periphery inwards, which in turn allows one to include it in an accurate detector model. Standoff distance has already been demonstrated with EXO-200 [10,5]. The sensitivity projections for nEXO shown in Fig. 5 are based on these studies.

Other nEXO-specific features include:

- Ionization charge readout pads with a finer 3-4 mm pitch (driven by EXO-200 data).

- Passive $\mathrm{Pb}$ shielding is substituted by a large water tank instrumented as a Čerenkov muon detector..

- Use of VUV-sensitive SiPM instead of LAAPDs. These will be installed on the cylindrical barrel, behind the voltage dividing field cage.

- Almost no plastics inside the TPC to help achieve $>10 \mathrm{~ms}$ electron drift lifetime $(\sim 5 \mathrm{~ms}$ demonstrated by EXO-200).

- Aluminization of optically passive surfaces to maximize scintillation light collection efficiency.

- In-xenon, cold readout electronics for scintillation and ionization.

- Installation deeper underground than WIPP (currently working closely with SNOLab, but also studying LNGS and Jinping sites for cosmogenic backgrounds, study based on EXO200 data).

\section{5. nEXO R\&D}

The $\mathrm{nEXO} R \& \mathrm{D}$ is progressing on the following fronts:

- Ultra-low background TPC design, with minimal use of plastics and aluminized, mirror-like surfaces. We are investigating the use of sapphire for mechanical structures, to substitute the acrylic used in EXO-200. The details of the aluminization are being investigated, along with possible flu ride coatings to prevent the aluminum from oxidising and losing its VUV reflectivity properties.

- Charge readout on metalled pads on quartz/sapphire supports instead of traditional crossed wires. This allows for a more compact design, and elimination of topology ambiguity for multi-site events.

- SiPMs instead of LAAPDs: SiPMs are quickly gaining the front stage as compact, low cost and low radioactivity light sensors for noble liquid detectors. We are developing VUVsensitive devices with several manufacturers [21].

- Investigation of carbon fiber for the cryostat.

- Development of low-noise, low-radioactivity cryogenic electronics for both the scintillation and ionization readout. The electronics needs to comply with stringent radioactivity, thermal dissipation, and noise requirements.

- Investigation of high voltage issues with noble liquid TPCs [22].

- Definition of traditional and innovative calibration strategies for a 5-tonne detector. 


\section{Acknowlegements}

EXO-200 and nEXO are supported by DoE and NSF (USA), NSERC (Canada), SNF (Switzerland), NRF (South Korea), RFBR (Russia), and the DFG Cluster of Excellence ÔUniverseÕ (Germany). A.P. acknowledges support from NSF grant PHY-1209907.

\section{References}

[1] S.R. Elliott and P. Vogel, Ann. Rev. Nucl. Part. Sci. 52 (2002), and references therein; F.T. Avignone III, S.R. Elliott, and J. Engel, Rev. Mod. Phys. 80, 481 (2008).

[2] J. Schechter and J.W.F. Valle, Phys. Rev. D25, 2951 (1982).

[3] M. Agostini et al., Phys. Rev. Lett. 111, 122503 (2013).

[4] A. Gando et al., Phys. Rev. Lett. 110, 062502 (2013).

[5] J. Albert et al., Nature 510, 229 (2014).

[6] H.V. Klapdor-Kleingrothaus and I.V. Krivosheina, Mod. Phys. Lett. A21, 1547 (2006).

[7] D.R. Artusa et al., Preprint arXiv:1402.6072 (2014).

[8] L. Sibley et al., AIP Conf.Proc. 1604, 449 (2014).

[9] N. Ackerman et al., Phys. Rev. Lett. 107, 212501 (2011).

[10] J.B. Albert et al., Phys. Rev. C89, 015502 (2013).

[11] M. Redshaw et al., Phys. Rev. Lett. 98, 053003 (2007).

[12] (EXO Collaboration) M. Auger et al., J. Inst. 7, P05010 (2012).

[13] M. Moe, Phys. Rev. C44, R931 (1991).

[14] (EXO-200 Collaboration) J. Albert et al., submitted by Phys. Rev. C, arXiv:1503.06241 (2015).

[15] (EXO-200 Collaboration) J. Albert et al., submitted to Phys. Rev. X, arXiv:1506.00317 (2015).

[16] EXO Collaboration, R. Neilson et el., Nucl. Instr. Meth A 608, 68 (2009).

[17] R. Luescher et al., Phys. Lett. B434, 407 (1998).

[18] E. Aprile et al., Phys. Rev. Lett. 107, 131302 (2011).

[19] D.S. Akerib et al., Phys. Rev. Lett. 112, 091303 (2014).

[20] (PandaX Collaboration) M. Xiao, et al., arXiv:1408.5114.

[21] I. Ostrovskiy et al., arXiv:1502.07837

[22] B. Rebel et al., J. Inst. 9, T08004 (2014). 